\title{
El efecto del absentismo universitario en el expediente académico y en la percepción de sus causas
}

University absenteeism effect in the academic performance and in its causes perception

\author{
Natividad Crespo Tejero (natividad.crespo@esic.edu) \\ $M^{a}$ Teresa Palomo Vadillo (maite.palomo@esic.es) \\ Mariano Méndez Suárez (mariano.mendez@esic.edu) \\ ESIC Business \& Marketing School de Madrid (España) \\ http://dx.doi.org/10.12795/EDUCADE.2012.103.04
}

RESUMEN: En este trabajo se presentan los resultados obtenidos en la evaluación de los motivos implicados en el absentismo en el Centro Universitario ESIC Business \& Marketing School. Para ello se ha utilizado una metodología cualitativa (Focus Group) y cuantitativa (cuestionario). Los resultados confirman la importancia de los factores relacionados con la cercanía de exámenes, la metodología del profesorado y los compromisos sociales. Además, el estudio aporta nuevas evidencias sobre la importancia de considerar las variables relacionadas con factores físicos, motivacionales y emocionales de los alumnos. Complementariamente el estudio evalúa los niveles de absentismo y confirma la estrecha relación con un inadecuado rendimiento académico.

PALABRAS CLAVE: Absentismo, Alumnos universitarios, Rendimiento académico.

\begin{abstract}
This paper presents the results obtained in the evaluation of the reasons involved in the absenteeism in the ESIC University Centre of Business and Marketing School. For that purpose, two techniques have been used: a qualitative (Focus Group) and a quantitative (questionnaire) methodology. The results confirm the importance of factors related to the nearness of examinations, the Professor's methodology and the social commitments. In addition, the study provides new evidences on the importance of considering variables related to physical, motivational and emotional factors that affect the students. In addition the study evaluates the levels of absenteeism and confirms the narrow relation with an inadequate academic performance.
\end{abstract}

KEYWORDS: Absenteeism, university students, academic performance.

\section{INTRODUCCIÓN}

En el ámbito universitario, el término absentismo ha sido definido como "la acción consciente y premeditada de ausentarse del medio físico de la clase universitaria, el aula, condicionada por una serie de factores, y que conduce a la búsqueda de alternativas en el uso del tiempo" (Del Moral, Navarro y García, 2010, p.20). Para la mejora de la calidad de la docencia y aumentar el rendimiento de los estudiantes se requiere que estos, asistan a clase con cierta normalidad. El absentismo en la educación se considera, por tanto, un problema de primer orden desde un punto de vista educacional, social, económico y personal. 
Entre los primeros estudios que analizan los niveles de asistencia a clase en la universidad, destaca la ampliamente citada investigación de Romer (1993). Los resultados del estudio señalan que un $33 \%$ de los alumnos de tres universidades americanas no asisten a clase. Por su parte, Marburger (2001) señala que el nivel de absentismo se sitúa alrededor del 18,5\%; mientras que Devadoss y Foltz (1996) encuentran una inasistencia a clase del $11 \%$ en cuatro universidades americanas. Más recientemente, Massingham y Herrington (2006) en Australia señalan que sólo el 16,2\% de los alumnos asiste a todas las clases presenciales y el $23,8 \%$ a todas las tutorías programadas.

En Europa, Timmins y Kaliszer (2002) evalúan el absentismo en estudiantes de enfermería de Irlanda. Señalan que en primer curso existe un $36 \%$ de episodios de absentismo, frente a un $48 \%$ en segundo curso y un $16 \%$ en tercer curso.

En España, destaca la investigación realizada por Rodríguez, Hernández, Alonso y DiezItza (2003) en la Universidad de Oviedo. Los resultados muestran que, un $40 \%$ de los sujetos asisten siempre a clase, mientras que un $51 \%$ lo hace con frecuencia y un $8,4 \%$ asisten sólo alguna vez. El estudio no encuentra diferencias significativas por sexo 0 curso; aunque los alumnos de primer curso muestran una peor asistencia. Posteriormente, el estudio de Álvarez et al. (2004) en la Universidad de Cádiz encuentra que el $75 \%$ de los alumnos asisten a más del $75 \%$ de las clases; un $20 \%$ asiste entre un $50 \%$ y $75 \%$ de las clases y sólo un $5 \%$ de los sujetos asisten por debajo del $50 \%$ de las clases. Tampoco señalan diferencias significativas en función del curso o titulación. Gracia y De la Iglesia (2007) realizan un estudio en la facultad de Económicas de la Universidad Complutense. Los análisis realizados revelan que el nivel de absentismo roza el 51,5\%. El estudio aporta diferencias entre sexos, obteniendo las chicas mejores resultados de asistencia. Por último, desde la Universidad de Vigo (Espada, 2008) se observa que un $67,5 \%$ de los alumnos informan asistir a clase siempre o con frecuencia; mientras que un $26,5 \%$ señala asistir alguna vez y, casi un $6 \%$ no asiste nunca. En este estudio, se observan diferencias significativas en función del sexo; y aunque no existen diferencias significativas en función del curso académico, sí aparecen peores resultados en primer curso, de forma similar a los resultados encontrados por Rodríguez et al. (2003). Es de destacar que los estudios disponibles se han realizado antes de la introducción de los nuevos grados en aplicación de los acuerdos de Bolonia. En este escenario no se considera obligatoria la asistencia a clase, mientras que en los Grados existe una mayor proporción de materias en las que se establece la obligatoriedad y/o evaluación de la asistencia, por lo tanto cabe esperar que con la implantación de los Grados disminuyan los niveles de absentismo.

A la luz de los estudios revisados podemos establecer que los niveles de inasistencia a clase son muy elevados, variando entre el $10 \%$ y el $35 \%$. Por otro lado, los estudios no son concluyentes sobre el impacto del género y del curso sobre el absentismo; aunque sí parecen señalar que los alumnos de primer curso y los estudiantes varones son los más proclives a padecer un mayor nivel de absentismo estudiantil universitario. Cabe destacar también la falta de una definición precisa (cuantitativamente) del concepto absentismo.

Estos elevados niveles de inasistencia a clase generan una gran preocupación en el ámbito educativo, debido al impacto que tiene el absentismo en el aprendizaje y rendimiento de los estudiantes. Este tema, ha sido ampliamente analizado desde diferentes estudios y, existe acuerdo en señalar una relación estadística positiva entre la asistencia a clase y el rendimiento académico de los alumnos (Devadoss y Foltz, 1996; Gracia y De la Iglesia, 2007; Lamdin, 1996; Marburger, 2006; Massingham y Herrington, 2006; Rodgers, 2001; Romer, 1993; Stanca, 2006). 
Por otro lado, el absentismo escolar se considera un precipitante del posterior abandono de los estudios universitarios, lo que genera a su vez un elevado coste económico, social y personal en nuestra sociedad. Así, diferentes informes señalan que la tasa de abandono de los estudios universitarios españoles se sitúa entre el $28 \%$ y $50 \%$ (Comunidad Escolar, 2006; Informe Global, 1996-2000; Informe CYD, 2008). Además, los mayores porcentajes de abandono se producen, con diferencia, por los alumnos de primer curso (Cabrera, Bethencourt, Álvarez y González, 2006).

Tras valorar en los estudios anteriores los niveles de absentismo, el siguiente paso será analizar las posibles causas del mismo, a fin de elaborar mejores estrategias para combatirlo.

La mayor parte de los estudios revisados están de acuerdo en la multicausalidad del problema del absentismo. Los primeros estudios que han analizado este aspecto se remontan a la década de los años 60, donde Dole y Digman (1967) en la universidad de Hawai muestran que existen 13 grandes grupos de razones para asistir a clase basadas en cuatro categorías: razones, intereses, valores e influencias. Clark (1975), por su parte, indica que la principal diferencia entre alumnos absentistas y no absentistas viene marcada por una ocupada vida social de los primeros.

En la década de los años 90, Devadoss y Foltz (1996) concluyen que los factores relacionados con la asistencia son: la motivación, la autofinanciación del alumno, las horas dedicadas al trabajo, la calidad del profesor, la nota media del curso anterior y el tipo de metodología utilizada en el aula.

Más recientemente, el estudio de Timmis y Kaliszer (2002) con una muestra de alumnos de enfermería en Irlanda identifica los compromisos sociales, personales y el estrés como los factores que determinan el absentismo universitario. Massingham y Herrington (2006) en Australia indican que los factores decisivos para no asistir a clase son: enfermedad, mostrarse ocupado, tener que trabajar, aguantar clases aburridas, poder conseguir los apuntes sin ir a clase, las tecnologías alternativas y el profesor. Finalmente, Doyle et al. (2008) encuentran que la insatisfacción con el profesor, el trayecto desde casa, los compromisos familiares y sociales, el estrés, etc., son motivos relacionados con el absentismo.

Ya en nuestro país, el estudio de Alvarez et al. (2004), señala que las principales causas de absentismo están relacionadas con la labor docente del profesor o con situaciones circunstanciales como la proximidad de exámenes. Otro estudio ampliamente citado es el realizado por Rodríguez et al. (2003). Si analizamos los factores que acumulan más de un $70 \%$ de acuerdo entre los alumnos, encontramos que "el profesor no tiene buena metodología" aparece señalando en primer lugar, seguido de "proximidad de exámenes" y en tercer lugar "el profesor no motiva". Posteriormente, Espada (2008) utilizando el mismo cuestionario de evaluación de causas del absentismo que Rodríguez et al. (2003) obtiene resultados prácticamente similares aunque, en este caso, el factor "proximidad de exámenes" aparece como el más señalado por los alumnos.

Recientemente, Triadó-Ivern, Aparicio-Chueca, Guàrdia-Olmos y Jaría Chacón (2009) y De Jorge, Gil, Merino y Sanz (2011) encuentran que los alumnos de la Universitat de Barcelona y Alcalá de Henares exponen como causas de absentismo variables externas a ellos, relacionadas fundamentalmente con la percepción de que las clases son pesadas y/o aburridas, bien por la materia en sí o por el propio profesor.

\subsection{OBjetivos}

En definitiva, los estudios analizados señalan que factores como los compromisos sociales, el trabajo, las clases aburridas, la inadecuada metodología del profesor y los 
exámenes próximos son manifestados por los alumnos universitarios, entre otros aspectos, como causas determinantes para no asistir a clase en un gran número de investigaciones.

Teniendo en cuenta las consideraciones anteriores, los objetivos del presente estudio consisten en evaluar el nivel de absentismo, relacionarlo con el rendimiento académico y analizar las principales causas de inasistencia a clase en alumnos universitarios. Para ello, hemos utilizado herramientas de investigación cualitativas y cuantitativas con el fin de obtener información directa de los alumnos y profesorado sobre los principales motivos que favorecen el absentismo universitario.

En concreto, nuestro estudio presenta las siguientes hipótesis:

a) Los motivos de inasistencia a clase serán diferentes entre alumnos absentistas y no absentistas.

b) Los alumnos con niveles elevados de absentismo universitario presentarán peores resultados académicos que los alumnos con una adecuada asistencia a clase.

\section{METODO}

\subsection{PARTICIPANTES}

La muestra incluye un total 545 alumnos procedentes del centro Universitario ESIC Business \& Marketing School. Del total 460 declararon su porcentaje de faltas y 225 además declararon su nota media en la carrera. Todas las titulaciones analizadas corresponden con carreras universitarias relacionadas con el área empresarial. La edad media de los participantes fue de 21,32 años, con una desviación típica de 1,90. La muestra incluye un porcentaje ligeramente superior de mujeres $(50,5 \%)$, frente al $49,5 \%$ de hombres.

\subsection{INSTRUMENTO}

Para la evaluación de las variables objeto de estudio se ha utilizado un cuestionario elaborado "ad hoc" formado por 77 ítems contestados sobre una escala tipo Likert de 5 niveles. El cuestionario recoge aquellos factores que estudios previos han considerado relevantes en el análisis del absentismo universitario. Además se han incluido otros factores obtenidos a través de grupos de discusión o "Focus Group", aplicados tanto a alumnos como a profesores (especialmente los primeros). Para potenciar la técnica, en nuestro estudio hemos utilizado el Diagrama de Ishikawa (Ishikawa, 1991), también conocido como diagrama causa-efecto o espina de pez. Este diagrama es una representación gráfica que muestra las relaciones entre una variable y los posibles factores desencadenantes o causales. Para construir el diagrama es necesario definir claramente el efecto (en nuestro estudio, el absentismo universitario) y posteriormente se insta a los participantes a señalar todas las posibles causas que puedan desencadenar el efecto. A modo de ejemplo, se incluye en la Figura 1 el diagrama de uno de los grupos de alumnos que han participado en la investigación.

En las sesiones de "Focus Group" de nuestro estudio han participado 180 alumnos de primero, segundo y tercer curso. Para el desarrollo de las sesiones se han formado grupos de máximo 6 alumnos, los cuales han generado, analizado y debatido las posibles causas del absentismo universitario. La información obtenida de los alumnos se completó con dos sesiones de "focus group" a quince profesores del centro. 
Figura 1. Diagrama de ISIKAWA

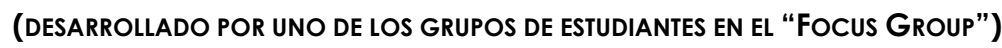

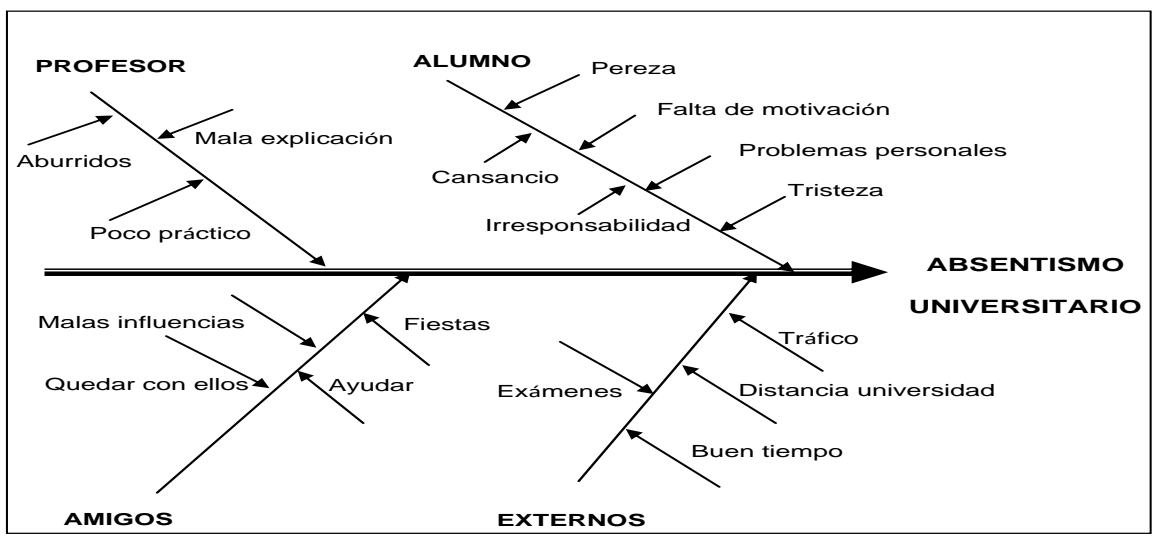

La gran cantidad de factores obtenidos a través del "focus group" exigió una reducción y clasificación de los mismos. En este sentido, los 118 ítems iniciales se redujeron a 77, que a su vez fueron clasificados por los investigadores en cuatro categorías principales y diez subcategorías. Las categorías incluyen:

- Factores relacionados con el/la alumno/a (factores motivacionales, problemas personales, familiares o sociales, estado físico y estado emocional).

- Factores relacionados con el profesorado (metodología y actitud).

- Factores relacionados con el entorno social (ocio).

- Factores relacionados con la universidad (organización, variables académicas y variables externas).

En el cuestionario utilizado para evaluar el absentismo se solicitan datos que corresponden al perfil del alumno/a, tales como edad, genero, curso, nota media, porcentaje de faltas, entre otros. El resto del cuestionario consta de 77 ítems en una escala tipo Likert (1, Muy en desacuerdo; 2, Algo de acuerdo; $3 \mathrm{Ni}$ de acuerdo ni en desacuerdo; 4, Muy de acuerdo; 5, Totalmente de acuerdo) que pretenden indicarnos si los alumnos están o no de acuerdo en que cada ítem particular influye en su decisión de no asistir a clase. En el anexo 1 se incluyen los elementos que conforman el cuestionario.

Para facilitar la comprensión del estudio hemos agrupado los ítems en función de las categorías desarrolladas.

\subsection{PROCEDiMiento}

Se trata de un estudio exploratorio y descriptivo transversal. Para llevar a cabo el estudio se efectuó un muestreo no probabilístico por conveniencia. El método utilizado ha sido cualitativo (Focus Group) y cuantitativo (cuestionario). La aplicación de la prueba se desarrolló durante las horas lectivas de clase. La entrega y recogida de los cuestionarios se llevo a cabo durante el mes de mayo de 2008. 


\section{RESULTADOS}

\subsection{PORCENTAJE DE faltAS}

En la Tabla 1 se puede observar el porcentaje de faltas de los 460 alumnos que lo declararon, en función del curso. Los alumnos indican si perciben faltar entre el 0 y $10 \%$ de las clases hasta un máximo de entre el 31 y el $40 \%$.

TABla 1. Porcentaje de faltas

\begin{tabular}{|c|c|c|c|c|c|c|c|c|}
\hline & \multicolumn{5}{|c|}{ Curso } & \multirow[b]{2}{*}{ Total } \\
\hline & & & 1 & 2 & 3 & 4 & 5 & \\
\hline \multirow{8}{*}{$\begin{array}{c}\% \\
\text { Faltas }\end{array}$} & \multirow{2}{*}{ Entre 0 y $10 \%$} & Recuento & 34 & 31 & 35 & 47 & 46 & 193 \\
\hline & & $\%$ del Curso & $38,6 \%$ & $31,3 \%$ & $38,5 \%$ & $48,0 \%$ & $54,8 \%$ & $42,0 \%$ \\
\hline & \multirow{2}{*}{ Entre 11 y $20 \%$} & Recuento & 32 & 38 & 36 & 33 & 27 & 166 \\
\hline & & $\%$ del Curso & $36,4 \%$ & $38,4 \%$ & $39,6 \%$ & $33,7 \%$ & $32,1 \%$ & $36,1 \%$ \\
\hline & \multirow{2}{*}{ Entre 21 y $30 \%$} & Recuento & 13 & 24 & 15 & 16 & 9 & 77 \\
\hline & & \% del Curso & $14,8 \%$ & $24,2 \%$ & $16,5 \%$ & $16,3 \%$ & $10,7 \%$ & $16,7 \%$ \\
\hline & \multirow{2}{*}{ Entre 31 y $40 \%$} & Recuento & 9 & 6 & 5 & 2 & 2 & 24 \\
\hline & & $\%$ del Curso & $10,2 \%$ & $6,1 \%$ & $5,5 \%$ & $2,0 \%$ & $2,4 \%$ & $5,2 \%$ \\
\hline & \multirow{2}{*}{ Total } & Recuento & 88 & 99 & 91 & 98 & 84 & 460 \\
\hline & & $\%$ del Curso & $100,0 \%$ & $100,0 \%$ & $100,0 \%$ & $100,0 \%$ & $100,0 \%$ & $100,0 \%$ \\
\hline
\end{tabular}

Podemos observar que en $4^{\circ}$ y $5^{\circ}$ cursos el porcentaje de alumnos que declaran entre 0 y $10 \%$ de faltas es de un $48 \%$ y un $54 \%$, dato muy superior al declarado por los alumnos de $1^{\circ}, 2^{\circ}$, y $3^{\circ}$, estando este entorno al $35 \%$. Podríamos considerar a partir de este dato que hay una disminución importante del absentismo a partir de $4^{\circ}$ curso.

\subsection{Clasificación alumnos abSentistas no abSentistas}

Con el objetivo de poder clasificar a los alumnos en función de su propensión al absentismo, se ha tomado como criterio el considerar "No absentistas" a los alumnos que declaran haber faltado a menos de un $20 \%$ de las clases, y considerar como "Absentistas" a los alumnos que declaren haber faltado en más de un $20 \%$ de las ocasiones.

A partir de los datos de la Tabla 2 se observa que el número de alumnos absentistas va disminuyendo progresivamente desde valores de un $25 \%$ en $1^{\circ}$, un $30 \%$ en $2^{\circ}$ y un $21,9 \%$ en $3^{\circ}$, hasta los valores de un $18 \%$ en $4^{\circ}$ y un $13 \%$ en $5^{\circ}$. 
TABla 2. Porcentaje de faltas entre alumnos abSentistas y alumnos no abSentistas

\begin{tabular}{|c|c|c|c|c|c|c|c|c|}
\hline & \multicolumn{5}{|c|}{ Curso } & Total \\
\hline \multirow{4}{*}{$\%$ Faltas } & Menos de un $20 \%$ & Recuento & 66 & 69 & 71 & 80 & 73 & 359 \\
\hline & & $\%$ de Curso & $75,0 \%$ & $69,7 \%$ & $78,0 \%$ & $81,6 \%$ & $86,9 \%$ & $78,0 \%$ \\
\hline & Más de un $20 \%$ & Recuento & 22 & 30 & 20 & 18 & 11 & 101 \\
\hline & & $\%$ de Curso & $25,0 \%$ & $30,3 \%$ & $22,0 \%$ & $18,4 \%$ & $13,1 \%$ & $22,0 \%$ \\
\hline
\end{tabular}

\subsection{RELACIÓN ENTRE ASISTENCIA Y RENDIMIENTO ACADÉMICO}

Para el análisis de la relación entre la nota media y el porcentaje de faltas se utilizan los datos aportados por 225 alumnos, que son los que declararon tanto su porcentaje de faltas como su nota media en la carrera.

Para establecer una relación funcional entre la asistencia a clase y el rendimiento académico se realizó una regresión en la que la variable independiente es el porcentaje de faltas y la variable dependiente la nota media en la carrera.

Como $\mathrm{H}_{0}$ : La asistencia no influye en el rendimiento académico y como $\mathrm{H}_{1}$ : La asistencia influye en el rendimiento académico.

Se obtuvieron los siguientes resultados, plasmados en la Tabla 3:

TABLA 3. Resultados de la Regresión ENTRE ASISTENCIA y PORCENTAJE de faltAS

\begin{tabular}{|c|c|c|c|c|}
\hline $\mathbf{R}^{\mathbf{2}}$ & $\mathbf{F}$ & & & \\
\hline 0,251 & $74,810^{* *}$ & & & \\
\hline \multicolumn{2}{|c|}{ ** Significativo al $99 \%$} & \multirow[b]{2}{*}{ Coeficientes } & \multirow[b]{2}{*}{ Error Típico } & \multirow[b]{2}{*}{$t$} \\
\hline & & & & \\
\hline \multicolumn{2}{|c|}{ Constante } & 7,824 & 0,148 & $52,818^{* *}$ \\
\hline \multicolumn{2}{|c|}{ Porcentaje de Faltas } & $-0,637$ & 0,074 & $-8,649 * *$ \\
\hline
\end{tabular}

Según los resultados podemos rechazar la Ho con más del $99 \%$ de confianza y afirmar que existe influencia del porcentaje de faltas en el rendimiento académico.

La relación funcional establecida es:

$$
\text { Nota Media }=7,82-0,63 \times \text { Porcentaje de Faltas }+e
$$


Es decir, un alumno que tuviera el $0 \%$ de faltas, en media, esperaría una nota de un 7,82 y paralelamente por cada $10 \%$ de aumento en las faltas esperaría una disminución de su nota de un 0,6.

Por otro lado el valor de $R^{2}$ o grado de explicación de las notas en función del porcentaje de faltas es de un $25 \%$, es decir, de todos los motivos que explican el expediente académico, la asistencia representaría un $25 \%$.

\subsection{RESULTADOS DE ÍTEMS CON MAYOR PUNTUACIÓN}

A continuación se indican los ítems a los que las respuestas de los alumnos otorgan una mayor importancia a la hora de decidir si asisten o no a clase. Se muestran en la Tabla 4 los ítems con una mediana mayor que 2 (Algo de Acuerdo):

TABLA 4. Medias, deSVIACIONES tíPICAS, MEDIANAS y MODAS DE LOS PRINCIPALES MOTIVOS DE ABSENTISMO

\begin{tabular}{|l|c|c|c|c|}
\hline & Media & $\begin{array}{l}\text { Desv. } \\
\text { típ. }\end{array}$ & Mediana & Moda \\
\hline $\begin{array}{l}\text { Tengo exámenes, presentaciones, trabajos de otras } \\
\text { asignaturas }\end{array}$ & 3.21 & 1.39 & 4 & 4 \\
\hline $\begin{array}{l}\text { Prefiero dedicar tiempo a estudiar, especialmente, cuando se } \\
\text { acerca la época de exámenes }\end{array}$ & 3.17 & 1.42 & 4 & 4 \\
\hline Me siento cansado/a & 2.95 & 1.37 & 3 & 4 \\
\hline Cuando hace buen tiempo, se está mejor fuera de clase & 2.94 & 1.40 & 3 & 2 \\
\hline Puedo decidir con libertad si asisto o no a clase & 2.81 & 1.55 & 3 & 1 \\
\hline Tengo planes alternativos de ocio: cafetería, deporte, etc. & 2.60 & 1.27 & 2 & 2 \\
\hline El/La profesor/a no explica con claridad & 2.56 & 1.18 & 2 & 2 \\
\hline Me da pereza ir a la universidad & 2.43 & 1.27 & 2 & 2 \\
\hline Tengo citas con mi pareja, amigos, etc. & 2.41 & 1.27 & 2 & 1 \\
\hline Las clases que recibo son monótonas y aburridas & 2.35 & 1.16 & 2 & 2 \\
\hline Las clases son muy teóricas & 2.32 & 1.14 & 2 & 1 \\
\hline $\begin{array}{l}\text { El profesorado muestra excesiva disciplina con la puntualidad } \\
\text { a primeras horas de clase }\end{array}$ & 2.29 & 1.33 & 2 & 1 \\
\hline Mis amigo/as me convencen para no entrar a clase & 2.28 & 1.22 & 2 & 1 \\
\hline $\begin{array}{l}\text { El/La profesor/a no se muestra accesible y abierto con los } \\
\text { alumnos }\end{array}$ & 2.25 & 1.15 & 2 & 1 \\
\hline El profesorado no motiva al alumnado & 2.23 & 1.15 & 2 & 1 \\
\hline $\begin{array}{l}\text { Tengo problemas con los medios de transporte para llegar } \\
\text { puntual a las clases }\end{array}$ & 2.18 & 1.38 & 2 & 1 \\
\hline $\begin{array}{l}\text { Me acuesto siempre tarde y, como consecuencia, tengo } \\
\text { sueño }\end{array}$ & 2.14 & 1.23 & 2 & 1 \\
\hline Las clases son muy seguidas, hay poco descanso entre clases & 2.11 & 1.25 & 2 & 1 \\
\hline El contenido de las asignaturas es muy fácil & 2.02 & 1.01 & 2 & 1 \\
\hline Cuando hace mal tiempo o llueve no me apetece ir a clase & 2.01 & 1.17 & 2 & 1 \\
\hline $\begin{array}{l}\text { El profesorado no se preocupa por hacer comprender sus } \\
\text { explicaciones }\end{array}$ & 2.00 & 1.06 & 2 & 1 \\
\hline El profesorado no aplica una buena metodología & 1.99 & 1.06 & 2 & 1 \\
\hline Mi estado de ánimo no es bueno: tristeza, apatía, etc. & 1.98 & 1.16 & 2 & 1 \\
\hline No se valora el esfuerzo del estudiante en el aula & 1.91 & 1.10 & 2 & 1 \\
\hline Simplemente, no estoy motivado & 1.91 & 1.12 & 2 & 1 \\
\hline $\begin{array}{l}\text { En clase no se transmite la relación entre el contenido de la } \\
\text { materia y sus posibles aplicaciones }\end{array}$ & 1.85 & 1.05 & 2 & 1 \\
\hline
\end{tabular}


Destacan dos ítems que corresponden a Variables Externas que están relacionados con presencia o cercanía de exámenes, son los que tienen una media mayor, por encima de 3 (Ni de acuerdo, ni en desacuerdo) y tienen una mediana y una moda igual a 4 (Muy de acuerdo).

En este caso y dada la importancia de este ítem para los alumnos, sería interesante el reflexionar desde el punto de vista docente, sobre la acumulación de pruebas y trabajos en fechas muy cercanas y averiguar hasta qué punto esta acumulación puede influir en la asistencia a clase en las últimas y cruciales fechas antes de los exámenes.

Previsiblemente con la implantación de lo Grados y de la evaluación continua estás aglomeraciones dejen de producirse.

A continuación le siguen tres ítems con mediana 3 y que corresponden a factores de Estado Físico, (Me siento cansado), otra vez de Variables Externas (Cuando hace buen tiempo...) y Factores Motivacionales (Puedo decidir con libertad.......).

El profesorado aparece por primera vez en séptimo lugar (El profesor no explica con claridad) con una mediana y moda de 2 (Algo de acuerdo), y en los puestos 10 y 11 con factores relacionados con la Metodología de impartición de clases (Clases monótonas..., Clases muy teóricas...) teniendo en ambos casos una mediana de 2.

\subsection{Resumen de LOS RESULtAdOS DE LOS CUESTIONARIOS}

Para intentar resumir los resultados se han clasificado las respuestas en 3 grupos en función de la importancia dada por los alumnos al ítem a la hora de decidir ir o no a clase. En concreto diferenciamos entre valores:

- Alto: Cuando se responde "Nada de Acuerdo" por un porcentaje de alumnos del $36 \%$ o inferior. El valor se interpreta como que este ítem tiene una influencia alta en la decisión de absentismo.

- Medio: Cuando se responde "Nada de Acuerdo" por un porcentaje de alumnos superior al $36 \%$ e inferior al $70 \%$. En este caso, el ítem influye de forma media en la decisión de absentismo.

- Bajo: Cuando se responde "Nada de Acuerdo" por un porcentaje de alumnos superior al 70\%. En este último caso se colige que el ítem no es relevante, o tiene una influencia muy baja a la hora de tomar la decisión de absentismo.

Debido a la extensión de los resultados se exponen a continuación únicamente las respuestas que han obtenido un porcentaje "alto" en el análisis realizado.

Complementariamente, se separan los porcentajes de respuestas entre alumnos considerados "No Absentistas (<20\% Faltas)" y los "Absentistas (>20\% Faltas)" con el objetivo de discernir si hay diferencia entre las opiniones sobre el ítem en cuestión entre los dos grupos.

La muestra de alumnos Absentistas consta de 101 alumnos y la de no Absentistas de 359 alumnos. A continuación se exponen los resultados agrupados por las categorías que han obtenido puntuaciones relevantes:

- Alumno. Factores Motivacionales. Dentro de este grupo de factores, que se pueden observar en la Figura 2, aquellos calificados con importancia alta son: "Puedo decidir con libertad si asisto o no a clase" y "Me da pereza ir a la universidad". 
FIGURA 2. DISTRIBUCIÓN DE FRECUENCIAS DE LOS ÍTEMS CONSIDERADOS DE ALTA INFLUENCIA EN LA SUBCATEgoría Factores MOtivacionales

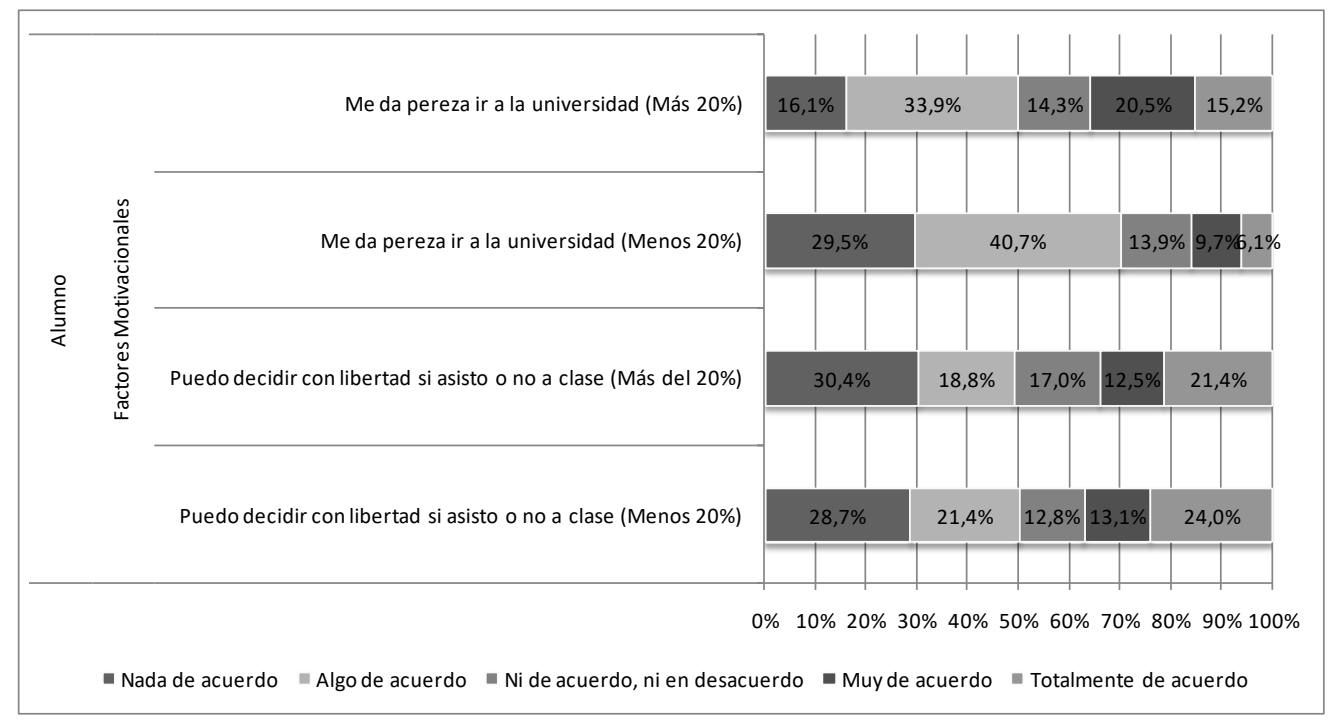

Si analizamos el ítem "Me da pereza ir a la Universidad" observamos que en el grupo de alumnos absentistas los que declararan "Nada de acuerdo" disminuye al 16,1\% y los que están "Muy de acuerdo" pasan a un 20,5\%. El grupo de los no absentistas mantiene su proporción, con lo que podríamos inferir que este aspecto influye más en los alumnos absentistas.

En el caso de "Puedo decidir con libertad si asisto a clase o no", se mantienen las proporciones con lo que consideramos que existe acuerdo en ambos grupos.

- Alumno. Estado Físico: En este caso destaca con importancia alta el ítem "Me siento cansado", donde se vuelven a observar diferencias entre ambos grupos, especialmente en los que responden "Nada de acuerdo" y "Algo de acuerdo". El resultado se puede observar en la Figura 3.

FiguRa 3. Distribución de fRECUENCIAS de LOS ÍtEMS CONSIDERADOS DE ALTA INFLUENCIA EN LA SUBCATEGORÍA ESTADO Fí́IICO

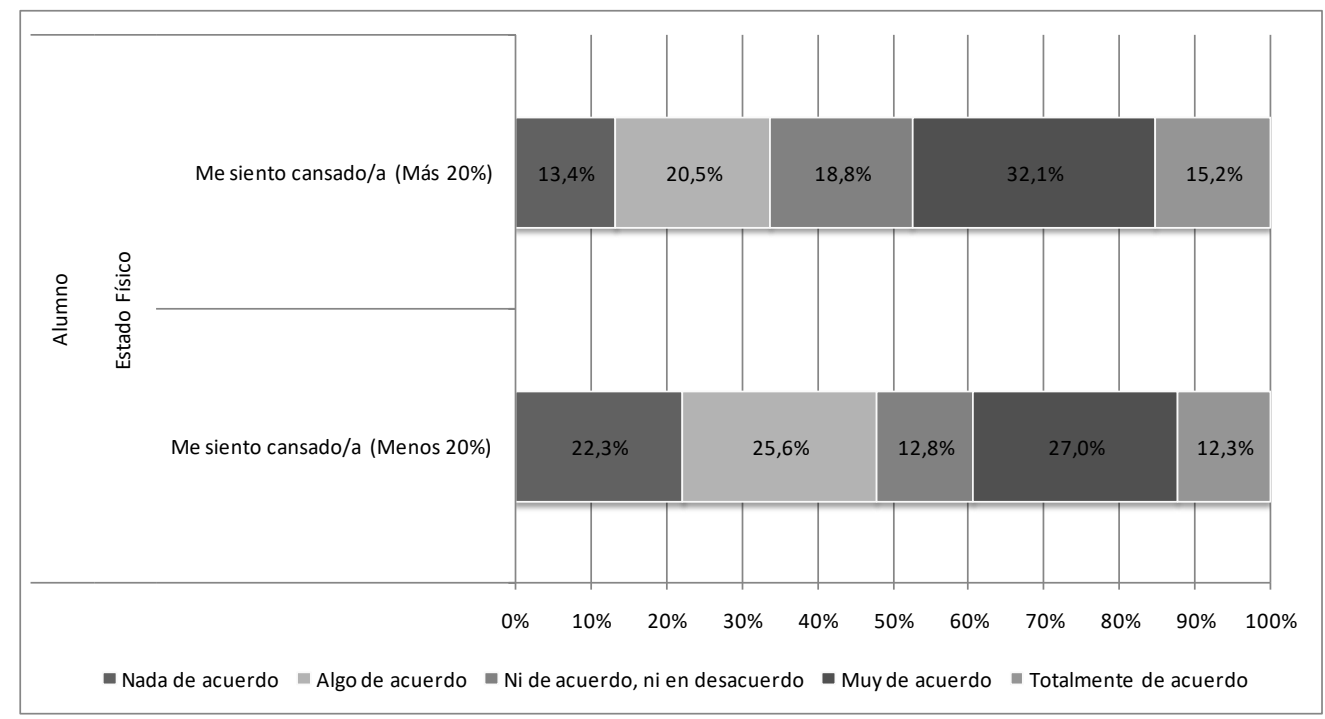

educade, $n$ ㅇ 3, 2012, p. 56 
- Profesorado. Metodología: Aquí aparecen tres factores con importancia alta, que son: "Las clases son muy teóricas", "Las clases que recibo son monótonas y aburridas" y "El profesor no explica con claridad".

En este caso hay una disminución del entorno de un $10 \%$ en el porcentaje de respuestas "Nada de acuerdo" de los alumnos absentistas respecto a los no absentistas, lo que podría indicar una mayor sensibilidad a la metodología docente de los alumnos absentistas. Los resultados aparecen en la Figura 4.

FiguRa 4. DistribUCIÓN DE FRECUENCIAS DE LOS ÍTEMS considerados de alta influencia en la subCategoría Metodología del Profesorado

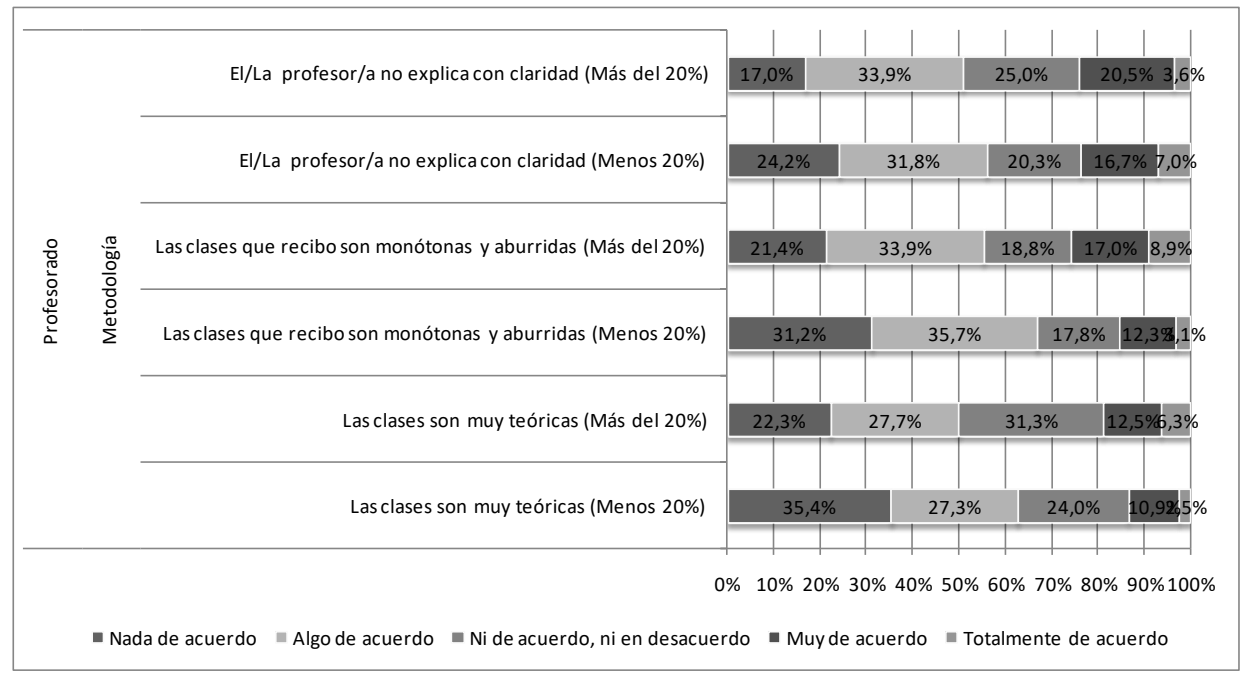

- Profesorado. Actitud: En esta categoría aparecen dos ítems con importancia alta que son "El profesorado no motiva al alumnado" y "El profesor no se muestra accesible y abierto con los alumnos". Al igual que en el caso anterior, y en este caso con diferencias en torno a un $16 \%$ en la respuesta "Nada de acuerdo", los alumnos absentistas son más sensibles a la actitud del profesorado en factores de motivación y accesibilidad al alumnado. Los resultados se encuentran en la Figura 5.

Figura 5. Distribución de fRECUencias de los ÍtemS Considerados de alta influencia en la subCategoría Actitud del Profesorado

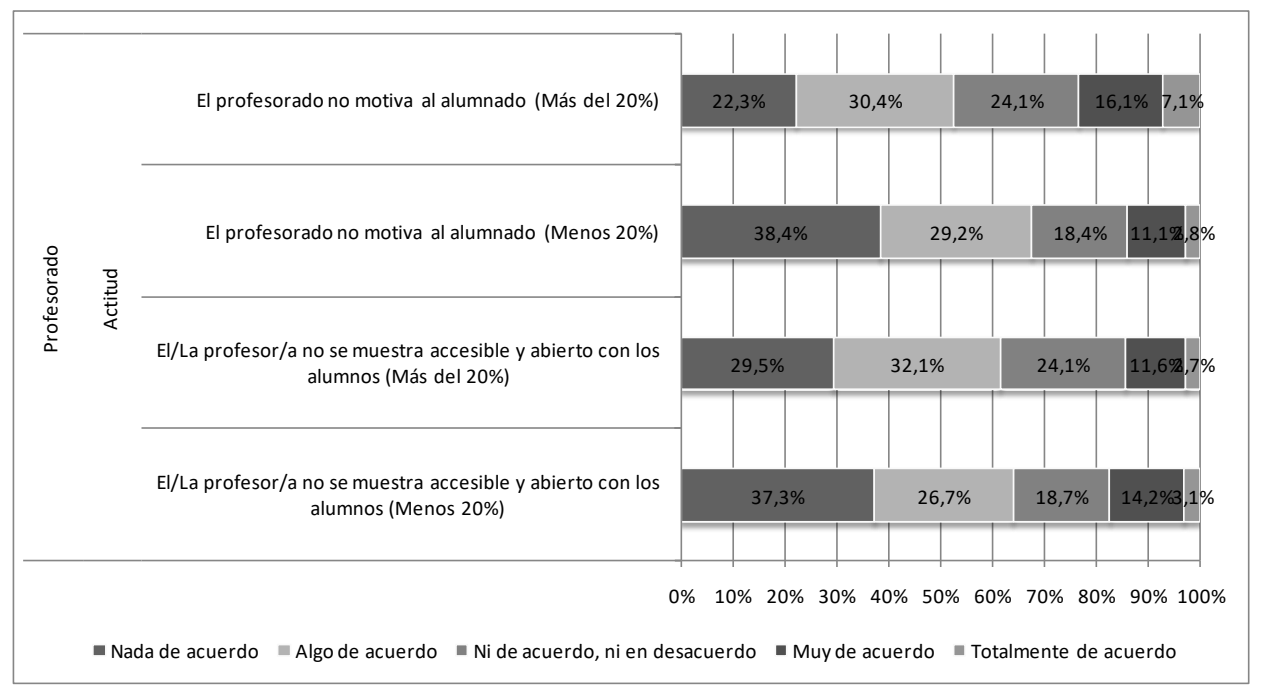


- Entorno Social. Ocio: En este grupo de factores destacan los ítems "Tengo planes alternativos de ocio", "Tengo citas con pareja, amigos, etc." y "Mis amigos me convencen para no entrar en clase". En este caso, las diferencias más significativas de entorno a un $10 \%$ en la respuesta "Nada de acuerdo" se da en todos los items. Como se puede observar en la Figura 6.

FiguRa 6. DistRIBUCIÓN DE FRECUENCIAS DE LOS ÍTEMS CONSIDERADOS DE ALTA INFLUENCIA EN LA SUBCATEGORÍA OCIO

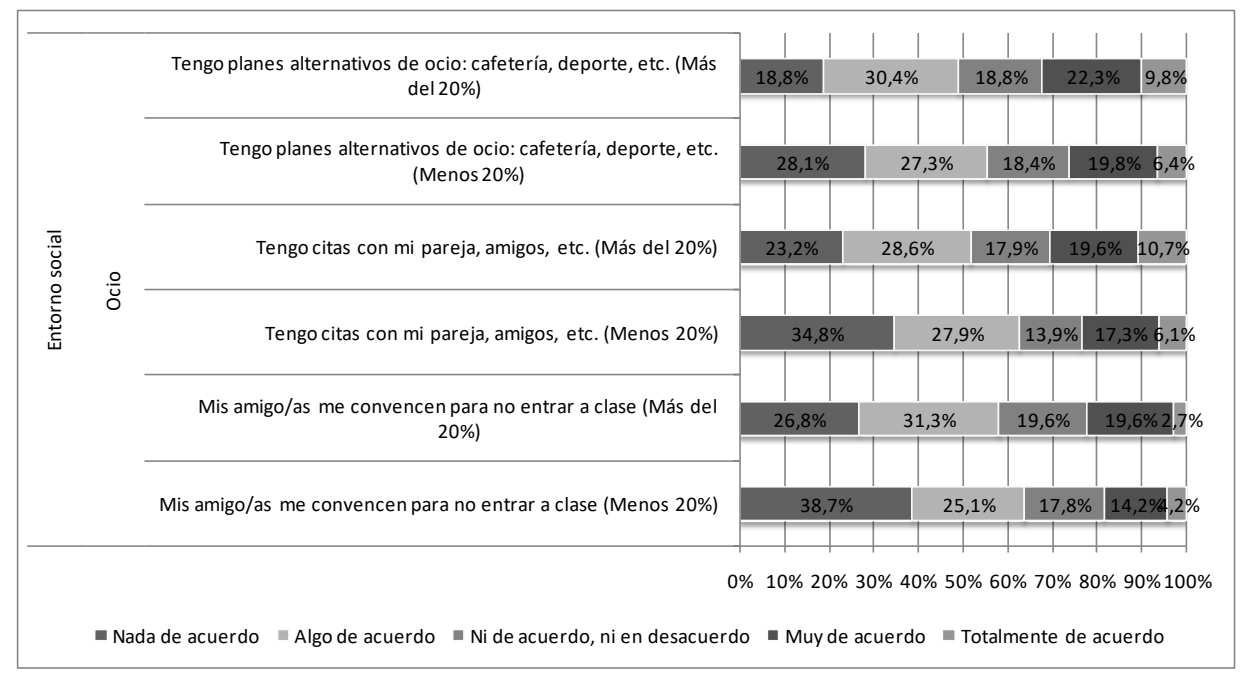

- Universidad. Variables Externas: Dentro de las variables consideradas como externas, destacan con importancia alta las referidas a: "Tengo exámenes, presentaciones, trabajos de otras asignaturas", "Prefiero dedicar tiempo a estudiar, especialmente, cuando se acerca la época de exámenes" y "Cuando hace buen tiempo, se está mejor fuera de clase". En este caso las diferencias en la respuesta "Nada de acuerdo" se situan entorno a un $8 \%$ lo que indicaría un mayor consenso entre ambos grupos de alumnos. Es llamativo que, excepto en el item "Tengo exámenes, presentaciones...", hay una diferencia del entorno del $10 \%$ más en las respuestas "Totalmente de acuerdo" de los alumnos absentistas. La diferencia es observable en la Figura 7.

FiguRa 7. DistribuCión de fRECUENCIAS DE LOS ÍTEMS CONSIDERADOS DE ALTA INFLUENCIA EN LA SUBCATEGORÍA VARIABLES EXTERNAS

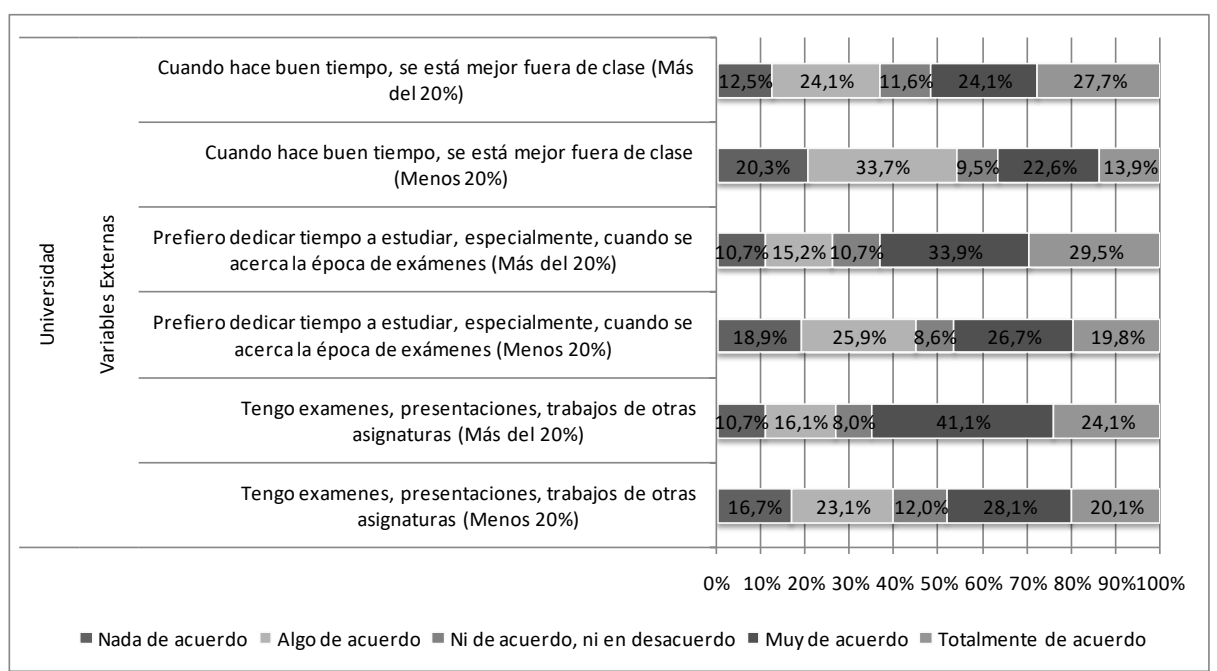

educade, no 3, 2012, p. 58 


\section{DISCUSIÓN}

El propósito de este trabajo ha sido analizar el problema del absentismo en alumnos universitarios. Para ello, además de estudiar la falta de asistencia a clase y su relación con el rendimiento académico, hemos llevado a cabo un análisis de los factores que inciden en el absentismo universitario.

Los resultados obtenidos en el análisis de la inasistencia a clase muestran que un $42 \%$ de los alumnos asiste a clase con regularidad (declaran faltar entre $0 \%$ y $10 \%$ de las clases), un $36 \%$ de estudiantes señalan no asistir entre un $11 \%$ y $20 \%$ de las clases; mientras que un $22 \%$ de alumnos declaran faltar a clase entre un $21 \%$ y $40 \%$. Estos datos son similares a los encontrados en estudios previos (Álvarez et al., 2004; Espada, 2008; Rodríguez et al., 2003) y confirman el alcance del absentismo universitario. Además, nuestro estudio muestra que a partir de tercer curso disminuye progresivamente el número de alumnos absentistas.

El análisis de la influencia del porcentaje de faltas sobre el rendimiento académico, muestra en consonancia con estudios previos una relación positiva (Devadoss y Foltz, 1996; Gracia y De la Iglesia, 2007; Lamdin, 1996; Marburger, 2006; Massingham y Herrington, 2006; Rodgers, 2001; Romer, 1993; Stanca, 2006).

Hasta el momento, la mayor parte de los estudios realizados han analizado los factores relacionados con el absentismo teniendo en cuenta a todos los alumnos que se encontraban en clase en el momento de la evaluación. Desde nuestro punto de vista, parece insuficiente generalizar resultados con el absentismo cuando quizás los alumnos que han contestado a los cuestionarios son estudiantes que habitualmente asisten a clase. Por ello, creemos adecuado que, además de analizar las variables que todos los alumnos manifiestan a la hora de faltar a clase, interesarnos por aquellos factores que afectan mayoritariamente a los alumnos absentistas.

Las variables que han obtenido las puntuaciones más elevadas en el cuestionario de absentismo se incluyen dentro de la subcategoría de "Variables externas". En concreto, al analizar las respuestas dadas por todos los alumnos (absentistas y no absentistas) encontramos que, en primer y segundo lugar respectivamente aparecen los ítems "Tengo exámenes, presentaciones o trabajos de otras asignaturas" y "Prefiero dedicar tiempo a estudiar, especialmente en época de exámenes". Además, al comparar la frecuencia de respuestas entre los alumnos absentistas y no absentistas encontramos importantes diferencias entre ambos grupos. Por ejemplo, un $63,4 \%$ de los alumnos absentistas señalan estar "Muy de acuerdo o totalmente de acuerdo" con el ítem "Cercanía de exámenes" como causa de la inasistencia a clase, frente al $46,5 \%$ de alumnos no absentistas. Estos datos muestran que la cercanía a los exámenes es considerado por los alumnos como un factor que influye de modo significativo en su decisión de no asistir a clase. Este dato es corroborado por diferentes estudios previos (Álvarez et al., 2004; Espada, 2008; Rodríguez et al, 2003). Sin duda, la tendencia del alumnado a aprovechar el tiempo de estudio en los momentos o días previos a la realización de un examen o a la presentación de un trabajo podría tener una lectura relacionada con una inadecuada gestión del tiempo, falta de organización o incluso un problema relacionado con la procrastinación o hábito de postergar actividades o situaciones que deben atenderse, sustituyéndolas por otras situaciones más irrelevantes y agradables. Por último, es interesante destacar que la cercanía de exámenes es un factor puntual y circunstancial. Este hecho invita a prestar atención a otros factores que puedan desarrollar su papel de manera más habitual en el absentismo de los alumnos.

Continuando con las variables externas, cabe destacar el ítem "Cuando hace buen tiempo, se está mejor fuera de clase". De hecho, este factor aparece como el cuarto 
elemento en orden de importancia en las respuestas dadas por todos los alumnos, sólo por detrás de los ítems "Cercanía de exámenes" y "Me siento cansado". También encontramos diferencias al dividir a los alumnos en absentistas y no absentistas. Así, sólo un $14 \%$ de los alumnos no absentistas están totalmente de acuerdo en considerar el buen tiempo como un factor que predice su posible inasistencia a clase, frente al doble $(27,7 \%)$ de los alumnos absentistas. Además de considerarlo un fenómeno externo e impredecible, no consideramos que sea un factor que por sí solo determine la inasistencia a clase, pero quizás si lo unimos con factores relacionados con la inadecuada metodología del docente o la influencia de los amigos, podría facilitar la decisión final de no asistir a clase.

Si nos centramos en la metodología del profesorado, este factor ha sido analizado en diferentes investigaciones previas con resultados concluyentes sobre la importancia de esta variable (Álvarez et at, 2004; De Jorge et al, 2011; Espada, 2008; Massingham y Herrington, 2006; Rodriguez et al, 2003; Triadó-Ivern et al., 2009). En nuestro estudio, se confirma la relevancia de los factores metodológicos analizados, aunque sorprende que éstos no aparezcan en los primeros puestos de importancia, como sucede en otros estudios. A pesar de ello, los ítems "Las clases son muy teóricas", "Las clases que recibo son monótonas y aburridas" y "El profesor no explica con claridad" son señalados reiteradamente por los alumnos para explicar su absentismo. Además, al diferenciar las respuestas dadas por los alumnos absentistas observamos que existe una disminución alrededor del $10 \%$ en el porcentaje de respuestas "Nada de acuerdo" de los alumnos absentistas respecto a los no absentistas en los ítems anteriormente citados.

En cuanto a la actitud que el alumno percibe del profesor, este factor ha sido por lo general, menos estudiado. Nuestros resultados muestran que los ítems "El profesor no se muestra accesible y abierto con los alumnos" y "El profesorado no motiva al alumnado" obtienen una importancia alta en el análisis realizado. En concreto, la percepción del alumnado sobre la falta de motivación por parte del profesorado parece más importante en el caso de los alumnos absentistas.

En definitiva, el análisis de todo el alumnado indica la importancia de los factores metodológicos $y$, en menor medida, de los factores relacionados con la actitud del profesorado. Además, los alumnos absentistas podrían manifestar una mayor sensibilidad a una metodología teórica, monótona o aburrida del profesorado y asimismo, "necesitar" una mayor motivación por parte de los docentes.

La revisión de la literatura sobre el absentismo universitario en nuestro país nos muestra que los factores analizados han sido considerados principalmente desde el punto de vista del profesor, centrándose en la metodología del mismo. Sin embargo, los modelos explicativos del absentismo laboral sí que conceden especial relevancia a factores relacionados con el propio empleado. Destacan factores como la motivación del individuo y su percepción de capacidad de asistencia (Rhodes y Steers, 1990), la satisfacción, actitudes, mecanismos de decisión, patrones de ausencia, etc. (Harrison y Martocchio, 1998). Por ello, nuestro estudio pretende obtener un punto de vista más amplio, incluyendo de modo relevante la variable "alumno". En este sentido, y después de constatar en los grupos de discusión que los propios estudiantes consideraban que ellos eran parte relevante del problema del absentismo, hemos querido analizar los factores físicos, motivacionales y emocionales de los individuos, los cuales, consideramos esenciales en los mecanismos de decisión del alumno.

A nivel motivacional, el análisis de la muestra de todos los alumnos (absentistas y no absentistas) subraya la importancia de los ítems "Me da pereza ir a la universidad" y "Puedo decidir con libertad si asisto o no a clase". De manera adicional, el análisis de las respuestas de los alumnos absentistas frente a los no absentistas nos muestra que los 
absentistas informan estar "muy de acuerdo o totalmente de acuerdo" en faltar a clase por "pereza" en una proporción que dobla a las contestaciones dadas por los alumnos no absentistas.

Si analizamos las variables que evalúan el estado físico de los alumnos, nos sorprende el destacado puesto (tercer lugar en las respuestas de los alumnos) que ocupa el ítem "Me siento cansado", sólo por detrás de la presencia de exámenes o trabajos cercanos. Al comparar los alumnos que asisten o no a clase con asiduidad encontramos diferencias entre ambos grupos, fundamentalmente en aquellos que responden "Nada de acuerdo" y "Algo de acuerdo".

En cuanto a los factores que estudian la incidencia del estado emocional de los alumnos en su decisión de no acudir a clase, podemos señalar que la mayor parte de los mismos reciben puntuaciones medias según los datos analizados. Cabe señalar que el primer factor emocional señalado por los alumnos ha sido "Mi estado de ánimo no es bueno: tristeza, apatía, etc.".

Por último, podemos descartar la subcategoría de problemas personales, familiares y sociales, como factores que fomenten el absentismo.

En resumen, nuestros resultados en relación con la variable alumno muestran que determinados factores motivacionales ("Me da pereza ir a la universidad"), factores físicos ("Me siento cansado") y, en menor medida, factores emocionales ("Mi estado de ánimo no es bueno...") son variables que caracterizan a los alumnos absentistas.

La importancia del entorno social del alumno en el absentismo universitario se confirma en nuestra investigación. Estos resultados apoyan estudios previos (Clark, 1975; Doyle et al., 2008; Timmis y Kaleszer, 2002). Así, factores como "Tengo planes alternativos de ocio", "Tengo citas con mi pareja, amigos, etc." y "Mis amigos me convencen para no entrar en clase" obtienen una elevada significación en el estudio. Además, al diferenciar entre alumnos absentistas y no absentistas encontramos que los primeros podrían manifestar un comportamiento más influenciable por el entorno social de amigos, compañeros, etc.

En definitiva, nuestro trabajo ha intentado ofrecer una visión multicausal del problema del absentismo. En este sentido, nuestros resultados confirman lo que han encontrado otros investigadores en relación con los factores externos al alumno, como la cercanía de exámenes, el buen tiempo, la inadecuada metodología del profesor, etc., y además muestran otros factores apenas considerados anteriormente.

El hecho de haber utilizado una técnica cualitativa como es el "Focus Group" para elaborar el cuestionario de absentismo, ha permitido considerar nuevos ítems, mayoritariamente asociados a la variable alumno. Resulta llamativa la relevancia de alguno de estos factores, incluso por delante de variables tan frecuentemente señaladas en estudios previos como la metodología del profesorado.

En último término, la elevada prevalencia del absentismo universitario y su relación con los exiguos resultados académicos nos lleva a intentar mejorar los factores que han demostrado su relación con este problema. Por un lado, debemos reflexionar sobre el papel del profesor en la educación universitaria. La forma de explicar, la falta de enfoque práctico de los contenidos teóricos, la necesidad de impartir clases dinámicas y ajustadas a los cambios que se desarrollan en nuestra sociedad son factores que los profesores debemos mejorar día a día en nuestra labor como docentes. Pero también hemos de considerar los factores dependientes del alumnado. Causas como cansancio, pereza o poco sentido de la responsabilidad aparecen de manera significativa. En este sentido, mejorar competencias personales del alumno a través de estrategias que favorezcan la gestión del tiempo, la motivación, el fomento de la responsabilidad, la seguridad en sí mismo, la madurez, 
etc., podrían favorecer el estado físico, motivacional y emocional del alumno y por ello obtener un mejor seguimiento de las clases académicas.

\section{LIMITACIONES Y CONSIDERACIONES SOBRE FUTUROS ESTUDIOS}

El hecho de haber realizado el estudio en horas lectivas puede introducir un pequeño sesgo en la obtención de los resultados, dado que aunque los estudiantes declaran su porcentaje de faltas, algunos de ellos y especialmente los absentistas es probable que ese día no hubieran asistido a clase.

Teniendo en cuenta las consideraciones anteriores, sería interesante la inclusión en futuros estudios de la relación entre absentismo y abandono, que puede ser una de las causas por la que reducen los niveles de absentismo en cursos superiores.

Por otro lado, sería interesante analizar los niveles de absentismo después de la introducción de los Grados.

\section{AGRADECIMIENTOS}

Queremos agradecer el apoyo y la colaboración del Profesor Ignacio Soret, el interesante análisis aportado por Macarena Estévez y los valiosos comentarios aportados en la evaluación anónima.

\section{BIBLIOGRAFÍA}

Álvarez, J. A., Duque, E., García, M., García, M.V., García, C., de Ory, I. y Rodríguez, I. (2004): Causas de la falta de asistencia a clase en una facultad de ciencias: análisis de los resultados de encuestas personales. Revista Española de Orientación y Psicopedagogía, 15(2): 317-335.

CABrera, L., Bethencourt, J.T., Álvarez, P. y GonzÁlez, M. (2006): El problema del abandono de los estudios universitarios. RELIEVE, 12(2): 171-203. Documento consultado en mayo de 2011, en: http://www.uv.es/RELIEVE/v12n2/RELIEVEv12n2_1.htm

CLARK, J. M. (1975): Time Out? A study of absenteeism among nurses. Londres: Royal College of Nursing.

COMUNIDAD ESCOLAR (2006): Información. Informe educativo de la Comisión Europea. Documento consultado en Abril de 2011, en: http://comunidadescolar.pntic.mes.es/789/info6.html.

De Jorge, J., GIL, L., Merino, F. y Sanz, M. (2011): Evidencia empírica de los motivos del absentismo en los estudiantes universitarios. Revista de Investigación en Educación, 9(2): 76-90.

Del Moral, L., Navarro, L. y García, M. (2010): Absentismo estudiantil en la Escuela Universitaria de Estudios Empresarial de la Universidad de Sevilla. En J.L. Jiménez y A. Rodríguez (Coords.): El absentismo en las aulas universitarias. El caso de la escuela universitaria de estudios empresariales de la Universidad de Sevilla, pp. 13-98. Sevilla: Grupo Editorial Universitario.

DEVADOSS, S. Y FOLTZ, J. (1996): Evaluation of factors influencing student class attendance and performance. American Journal of Agriculture Economics, 78(3): 499-507.

Dole, A.A. Y DIGMAN, J.M. (1967): Factors in college attendance. Journal of Applied Psychology, 51 (3): 247-253.

DOYLE, L., O`BRIEN, F., TIMMINS, F., TOBIN, G., O`ROURKE, F. Y DOHERTY L. (2008): An evaluation of an attendance monitoring system for undergraduate nursing students. Nurse Education in Practice, 8(2): 129-139.

ESPADA, L. (2008): El absentismo estudiantil universitario. Comunicación presentada en: Conferencia Estatal de Defensores Universitarios. Oviedo. España. Documento 
consultado en septiembre de 2011, en:

http://www.cedu.es/files/absentismoestudiantil.pdf.

Gracia, E. Y DE LA IGLESIA, M.C. (2007): Absentismo y resultados de los alumnos. Investigaciones de Economía de la Educación, 2: 219-30.

HARRISON, D.A. Y MARTOCCHIO, J.J. (1998): Time of absenteeism: A 20-year review of origins, offshoots, and outcomes. Journal of Management, 24(3): 305-350.

INFORME GlOBAL (1996-2000): Elaborado por el Consejo de Coordinación Universitaria. Documento consultado en junio de 2011, en: http://comunidadescolar.cnice.mec.es/718/univerl.html.

INFORME CYD (2008): Ministerio de Ciencia e Innovación y la fundación conocimiento y desarrollo (CYD). Documento consultado en julio de 2011, en: http://fundacioncyd.org.

ISHIKAWA, K. (1991): ¿¿Qué es el control total de calidad?: la modalidad japonesa. Bogotá: Norma.

LAMDIN, D.J. (1996): Evidence of student attendance as an independent variable in education production functions. The Journal of Educational Research, 89(3): $155-162$.

MARBURGER, D.R. (2001): Absenteeism and undergraduate exam performance. Journal of Economic Education, 32: 99-109.

MARBURGER, D.R. (2006): Does mandatory attendance improve student performance?. Journal of Economic Education, 37(2): 148-155.

MASSINGHAM, P. Y HERRINGTON, T. (2006): Does Attendance Matter? An Examination of Student Attitudes, Participation, Performance and Attendance. Journal of University Teaching \& Learning Practice, 3(2): 83-103.

RHODES, S. R. Y STEERS, R.M. (1990): Managing employee absenteeism. Indiana: Addison Wesley.

Rodríguez, R., Hernández, J., Alonso, A. y Diez-ItzA, E. (2003): El absentismo en la Universidad: resultados de una encuesta sobre motivos que señalan los estudiantes para no asistir a clase. Aula Abierta, 82: 117-145.

RODGERS, J.R. (2001): A panel-data study of the effect of student attendance on university performance. Australian Journal of Education, 45(3), 284-295.

ROMER, D. (1993): Do students go to class? Should they?. Journal of Economic Perpectives, 7: 167-174.

STANCA, L. (2006): The effects of attendance on academic performance: panel data evidence for introductory microeconomics. Journal of Economic Education, 37(3): 251-266.

TIMMINS, F. Y KALISZER, M. (2002): Absenteeism among nursing students - fact or fiction?. Journal of Nursing Management, 10(5): 251-264.

Triadó-IVern, X., Aparicio-ChueCA, P., GuÀrdia-Olmos, J. y Jaría-Chacón, N. (2009): Aproximación empírica al análisis del absentismo de los estudiantes universitarios. Estudio del caso de la Facultad de Economía y Empresa (UB). Revista de Formación e Innovación Educativa Universitaria, 2(4): 182-192. 


\section{Anexo 1. Cuestionario de absentismo}

"Te solicitamos que señales en qué medida cada uno de los siguientes elementos puede jugar un papel importante en la decisión de NO asistir a clase. Es decir, decido no asistir a clase porque. ...Por favor, contesta con sinceridad y piensa en las causas reales."

\section{Alumno}

- Factores Motivacionales: No tengo interés hacia los estudios que realizo / Simplemente, no estoy motivado/ Todo me aburre/ Estoy convencido/a que "sin ir a clase se aprueba"/Me comporto de forma irresponsable/ Puedo decidir con libertad si asisto o no a clase/Mi situación socioeconómica me permite no preocuparme por mi futuro profesional/Tengo todas mis necesidades cubiertas, da igual si hago o no una carrera/Si te obligan a ir a clase, "haces lo contrario"/Elegí mal la carrera/Seguir el ritmo de la clase implica demasiado esfuerzo/No tengo habito de asistir a clase/No tengo hábitos de puntualidad/Mi carrera universitaria no es prioritaria para mi/Prefiero estudiar por apuntes que ir a clase/Por costumbre falto a las primeras y/o ultimas horas del día/No encuentro una relación directa entre los contenidos de la asignatura y mis expectativas de formación/Me da pereza ir a la universidad/Prefiero asistir a una academia o clases particulares que ir a clase/He pensado en dejar de estudiar y empezar a trabajar/He pensado en cambiar de carrera.

- Problemas personales, familiares, sociales: Mi relación con el grupo de clase es insoportable/Tengo serios problemas personales/Tengo problemas familiares/ A mis padres sólo les interesa mi aprobado, el resto no les importa/Tengo problemas en mi relación de pareja.

- Estado Físico: Sufro problemas de salud/Me siento cansado/a/ Me acuesto siempre tarde y, como consecuencia, tengo sueño/Como no desayuno en casa, me voy a desayunar porque tengo hambre.

- Estado Emocional: Tengo problemas de ansiedad y/o estrés/Mi estado de ánimo no es bueno: tristeza, apatía, etc./No me siento seguro/ Me preocupa que se pregunte en clase/ No consigo adaptarme a este centro educativo/Creo que no puedo aprobar la asignatura.

\section{Profesorado}

- Metodología: El/La profesor/a no explica con claridad/ El profesorado no aplica una buena metodología/El profesorado no se preocupa por hacer comprender sus explicaciones/ El profesorado no fomenta la participación en clase/ Las clases que recibo son monótonas y aburridas/Las clases son muy teóricas/La asistencia no me facilita la comprensión de la materia/ Las explicaciones no se adaptan a mi nivel de conocimiento y comprensión/En clase no me transmite la relación entre el contenido de la materia y sus posibles aplicaciones.

- Actitud: El profesorado muestra excesiva disciplina con la puntualidad a primeras horas de clase/El profesorado no motiva al alumnado/El/La profesor/a no se muestra accesible y abierto con los alumnos/ El profesorado no se interesa por el alumnado/Tengo mala relación o conflictos con profesores/as/El profesorado demuestra falta de autoridad y de disciplina en el aula/En clase se enfatizan mis fracasos y se minimizan mis éxitos/No se valora el esfuerzo del estudiante en el aula/En ocasiones, se censura o se pone en evidencia a algún alumno

\section{Entorno Social}

- Ocio: Tengo planes alternativos de ocio: cafetería, deporte, etc./Salgo entre semana por la noche y al día siguiente no voy a clase/Tengo citas con mi pareja, amigos, etc./Mis amigo/as me convencen para no entrar a clase

\section{Universidad}

- Organización: Los horarios de las asignaturas de diferentes cursos se solapan/ Tengo demasiadas horas libres entre asignaturas/Las clases son muy seguidas, hay poco descanso entre clases/Se me dan facilidades como la plataforma on-line (apuntes, 
casos, etc.) que hacen que no pase nada si falto a clase/El sistema de control de faltas no se aplica de forma rigurosa/Las condiciones ambientales(temperatura, iluminación, ruido, etc.) de las aulas no son adecuadas/Los materiales empleados en el aula son poco adecuados.

- Académicas: El contenido de las asignaturas es muy fácil/El contenido de las asignaturas de mi curso son muy difíciles/La evaluación no se corresponde con lo explicado en el aula.

- Variables Externas: Cuando hace buen tiempo, se está mejor fuera de clase/Cuando hace mal tiempo o llueve no me apetece ir a clase/Tengo problemas con los medios de transporte (metro, autobús, coche, etc.) para llegar puntual a las clases/Tengo la obligación de realizar otras actividades: gestiones, banco, hacienda, etc./Doy prioridad a las prácticas en una empresa y mis estudios son secundarios/Mis padres ni me controlan, ni me obligan asistir a clase/Tengo que trabajar para contribuir a los gastos de mi familia/Prefiero dedicar tiempo a estudiar, especialmente, cuando se acerca la época de exámenes/Tengo exámenes, presentaciones, trabajos de otras asignaturas. 